УДК 504.064.2:502.2.08:551.4.044:528.854.2:519.876.5

ВЕЙВЛЕТ-АНАЛИЗ РАСПРЕДЕЛЕНИЯ ДОЛГОТЫ В ХАРАКТЕРНЫХ ТОЧКАХ ПРОДОЛЬНОГО ПРОФИЛЯ РУСЛА МАЛОЙ РЕКИ ИРОВКА

Мазуркин П.М., Георгиева Я.О.

Поволжский государственный технологический университет, Йошкар-Ола, e-mail:kaf_po@mail.ru,yanageorgieva2017@yandex.ru

Река Ировка по широте находится в прямоугольнике 23.89 мин и по долготе 18.89 мин. Цель статьи анализ волновых уравнений в виде асимметричных вейвлетов изменения координаты - местной долготы. После идентификации вейвлета были получены 18 составляющих с переменными амплитудой и периодом колебания. Значимая часть колебаний происходит вдоль р. Ировка протяженностью 69 км. Три первых члена дали корреляцию 0.9991, что чуть меньше для местной широты 0.9999. Уровень адекватности равен более 0.9 , и поэтому уравнение является сильнейшей факторной связью. Другие 15 членов повышают уровень адекватности до 1. Анализ показал, что местная долгота малой реки по измерениям на снимках из космоса дает высокую определенность квантованию колебаний. По интервалам погрешность моделей после 18 -го члена распределяется так: 0-1.0\% 203 шт. (70\%); от 0 до 5\% - 277 точек русла, что равно $95.52 \%$ от 290 характерных точек. Норму погрешности моделирования 5\%, тогда оставшиеся $290-277=13$ точек требуют отдельного анализа. Влияние широты на долготу показано двухчленным трендом и 11 волновыми уравнениями, и это сложнее известных рядов Фурье: амплитуда и период колебаний являются переменными. Резкие изменения кривизны русла в плане по характерным точкам не менее 10-150 дали добротный статистический материал. Заметна фрактальность рельефа и русла вдоль малой реки: чем точнее будут измерения ландшафта, тем больше будет выявлено вейвлетов.

Ключевые слова: малая река, космический снимок, русло, точки, долгота, распределения, вейвлеты

\title{
WAVELET ANALYSIS OF EAST LONGITUDE AT CHARACTERISTIC POINTS OF THE LONGITUDINAL PROFILE SMALL RIVER IROVKA
}

\author{
Mazurkin P.M., Georgieva Ya.O. \\ Volga State University of Technology, Yoshkar-Ola, \\ e-mail:kaf_po@mail.ru,yanageorgieva2017@yandex.ru
}

\begin{abstract}
The Irovka River in latitude is in the rectangle 23.89 minutes and in longitude 18.89 minutes. The purpose of the article is the analysis of wave equations in the form of asymmetric wavelets of coordinate change - local longitude. After identifying the wavelet, 18 components with variable amplitude and oscillation period were obtained. A significant part of the oscillations occurs along the Irovka River with a length of $69 \mathrm{~km}$. The first three members gave a correlation of 0.9991 , which is slightly less for the local latitude 0.9999 . The adequacy level is more than 0.9 and therefore the equation is the strongest factorial relationship. The other 15 members increase the level of adequacy to 1 . The analysis showed that the local longitude of a small river, as measured by images from cosmos, gives a high certainty to the quantization of oscillations. At intervals, the error of the models after the 18th term is distributed as follows: $0-1.0 \% 203$ pcs. $(70 \%$ ); from 0 to $5 \%-277$ points of the channel, which is $95.52 \%$ of 290 characteristic points. The norm of the modeling error is $5 \%$, then the remaining $290-277=13$ points require a separate analysis. The influence of latitude on longitude is shown by two-membered trend and 11 wave equations, and it is more complicated than the well-known Fourier series: the amplitude and period of oscillations are variable. The sharp changes in the curvature of the channel in terms of characteristic points of at least 10-150 yielded sound statistical data. The fractality of the relief and the channel along the small river is noticeable: the more accurate the measurements of the landscape, the more wavelets will be revealed.
\end{abstract}

Keywords: small river, satellite image, channel, points, longitude, distribution, wavelets

Одним из возможных путей анализа дистанционного зондирования Земли является использование подходов математической морфологии ландшафтов - направления ландшафтоведения, изучающего количественные закономерности построения мозаик, образованных на земной поверхности, и разрабатывающего методы их математического анализа [1].

В статье [2] дан краткий обзор опыта применения гармонического анализа для морфометрической характеристики рельефа. Получены до 10 гармоник с постоянными периодом и амплитудой колебания. Преобразование Фурье может использоваться для задач классификации и районирования земной поверхности по ее гармоническим характеристикам, определяющим специфику топографического расчленения участка. В другой статье [3] показано, что цифровые модели рельефа и космические снимки позволяют выявить важные характеристики ландшафтов применительно к элементам рельефа, проследить изменения и спрогнозировать последствия природно-антропогенного и антропогенного воздействия на экосистемы.

По космическим снимкам получены координаты (широта, долгота, высота) по ин- 
струкции [4]. На линии стрежня в русле малой реки выбираются характерные точки по космическому снимку от истока до устья и составляется таблица исходных данных для последующего статистического моделирования методом идентификации волновых закономерностей [5].

Цель - выявление и анализ асимметричных вейвлетов по одной координате - приведенной к параметрам р. Ировка местной восточной долготе. Для этого от истока до устья в русле стрежня Ировки по кривизне русла выделили 290 характерных точек. Методика измерений была показана в статье [6].

\section{Материалы и методы исследования}

В табл. 1 даны измерения и их сравнение с моделями.

Асимметричные вейвлет-сигналы записываются волновой формулой [5] вида

$$
\begin{gathered}
y_{i}=A_{i} \cos \left(\pi x / p_{i}-a_{8 i}\right), \\
A_{i}=a_{1 i} x^{a_{2 i}} \exp \left(-a_{3 i} x^{a_{4 i}}\right), \\
p_{i}=a_{5 i}+a_{6 i} x^{a_{7 i}},
\end{gathered}
$$

где $y$ - учитываемый показатель (зависимый фактор), $i$ - номер составляющей общей модели (1), $m$ - количество членов в модели (1), $x$ - объясняющая переменная (влияющий фактор), $a_{1} \ldots a_{8}-$ параметры модели (1), принимающие числовые значения в ходе структурно-параметрической идентификации в программной среде CurveExpert-1.40, $A_{i}$ - амплитуда (половина) вейвлета (ось $y), p_{i}-$ полупериод колебания (ось $x$ ).

\section{Результаты исследования и их обсуждение}

В табл. 2 приведены значения параметров модели (1) по 13 членам влияния местной широты на долготу.

На рис. 1 даны графики трех членов и их объединения в одну формулу.

Таким образом, распределение долготы от широты определяется волновыми уравнениями, причем они гораздо сложнее рядов Фурье, так как амплитуда и период колебаний переменные. Двухчленный тренд показывает, что малая река Ировка протекает в прямоугольнике 23.89 мин длиной (по местной широте Север - Юг) и 18.89 мин шириной (по местной долготе). Резкие изменения кривизны русла в плане по 290 характерным точкам дали 11 волновых уравнений (табл. 2). Таким образом, заметна фрактальность в строении рельефа и русла малой реки: чем точнее будут измерения ландшафта, тем больше будет выявлено вейвлетов.

Таблица 1

\begin{tabular}{|c|c|c|c|c|c|c|}
\hline \multirow{2}{*}{$\begin{array}{l}\text { Ранг } \\
\text { точки }\end{array}$} & \multirow{2}{*}{$\begin{array}{c}\text { Ранг } \\
R_{\beta}\end{array}$} & \multirow{2}{*}{$\begin{array}{c}\text { Восточная } \\
\text { долгота }\end{array}$} & \multirow{2}{*}{$\begin{array}{c}\text { Долгота } \\
\beta, \text { минута }\end{array}$} & \multicolumn{3}{|c|}{ Расчетные значения } \\
\hline & & & & $\beta$, минута & $\varepsilon_{18}$, минута & $\Delta, \%$ \\
\hline 0 & 20 & $\mathrm{E} 49^{\circ} 20.09^{\prime}$ & 17.39 & 17.425 & 0.0350129 & 0.20 \\
\hline 1 & 19 & $\mathrm{E}^{\prime} 9^{\circ} 20.20^{\prime}$ & 17.50 & 17.527 & 0.0268094 & 0.15 \\
\hline 2 & 17 & $\mathrm{E} 49^{\circ} 20.32^{\prime}$ & 17.62 & 17.611 & -0.00942622 & -0.05 \\
\hline 3 & 15 & $\mathrm{E} 49^{\circ} 20.37^{\prime}$ & 17.67 & 17.609 & -0.0605765 & -0.34 \\
\hline & & & & & & \\
\hline 250 & 287 & $\mathrm{E} 49^{\circ} 3.371^{\prime}$ & 0.230 & 0.307 & 0.0767206 & 33.36 \\
\hline$\ldots$ & $\ldots$ & & $\ldots$ & $\ldots$ & & 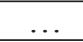 \\
\hline 287 & 171 & $\mathrm{E} 49^{\circ} 4.719^{\prime}$ & 2.019 & 2.010 & -0.00857075 & -0.42 \\
\hline 288 & 164 & $\mathrm{E} 49^{\circ} 4.735^{\prime}$ & 2.035 & 2.048 & 0.0134797 & 0.66 \\
\hline 289 & 172 & $\mathrm{E} 49^{\circ} 4.717^{\prime}$ & 2.017 & 2.032 & 0.0147019 & 0.73 \\
\hline
\end{tabular}

Ранговые распределения характерных точек и местной долготы продольного профиля русла малой реки Ировка

\begin{tabular}{|c|c|c|c|c|c|c|c|c|c|}
\hline \multirow{3}{*}{$\begin{array}{c}\text { Номер } \\
i\end{array}$} & \multicolumn{8}{|c|}{ Вейвлет $y_{i}=a_{1 i} x^{a_{2 i}} \exp \left(-a_{3 i} x^{a_{4 i}}\right) \cos \left(\pi x /\left(a_{5 i}+a_{6 i} x^{a_{7 i}}\right)-a_{8 i}\right)$} & \multirow{3}{*}{$\begin{array}{c}\text { Коэф. } \\
\text { корр. } \\
r\end{array}$} \\
\hline & \multicolumn{4}{|c|}{ Амплитуда (половина) колебания } & \multicolumn{3}{|c|}{ Полупериод колебания } & СдВиг & \\
\hline & $a_{1 i}$ & $a_{2 i}$ & $a_{3 i}$ & $a_{4 i}$ & $a_{5 i}$ & $a_{6 i}$ & $a_{7 i}$ & $a_{8 i}$ & \\
\hline 1 & 19.08116 & 0 & 0.039811 & 1.32388 & 0 & 0 & 0 & 0 & 0.9976 \\
\hline 2 & $-1.61185 e-6$ & 26.25390 & 14.89933 & 0.49461 & 0 & 0 & 0 & 0 & \\
\hline 3 & -2.09089 & 0 & 0.82090 & 0.40884 & 2.36913 & 0.0028086 & 1.55183 & -0.35993 & \\
\hline
\end{tabular}

Таблица 2

Влияние широты Север - Юг от истока до устья на долготу реки Ировка 


\begin{tabular}{|c|c|c|c|c|c|c|c|c|c|}
\hline & & & & & & & Око1 & нчание 1 & габл. 2 \\
\hline Номер & & Вейвле & $y_{i}=a_{1 i} x^{a_{2 i}}$ & $\mathrm{p}\left(-a_{3 i} x^{a_{4}}\right.$ & $\cos (\pi x /(a$ & $\left({ }_{5 i}+a_{6 i} x^{a_{7 i}}\right)$ & $\left.-a_{8 i}\right)$ & & Коэф. \\
\hline & Ампл & итуда (пол & овина) колеб & ания & Полуг & териод колес & бания & Сдвиг & \\
\hline & $a_{1 i}$ & $a_{2 i}$ & $a_{3 i}$ & $a_{4 i}$ & $a_{5 i}$ & $a_{6 i}$ & $a_{7 i}$ & $a_{8 i}$ & \\
\hline 4 & $1.95823 \mathrm{e}-5$ & 0 & 0.448432 & 1.00282 & 0.0820466 & 0.0119878 & 1.00090 & 5.45636 & 0.4873 \\
\hline 5 & 0.0168527 & 3.08551 & 0.378919 & 1.00055 & 1.58592 & $6.89209 \mathrm{e}-5$ & 2.25901 & 2.74470 & 0.5584 \\
\hline 6 & $4.51507 \mathrm{e}-6$ & 4.65846 & 0.00126702 & 2.65421 & 0.156256 & 0.232502 & \begin{tabular}{|l|}
0.219714 \\
\end{tabular} & 12.08418 & 0.3172 \\
\hline 7 & 0.00742864 & 0 & 2.07429 & 0.127857 & -0.0334208 & 1.99360 & 0.0795731 & 6.22674 & 0.3006 \\
\hline 8 & $1.14486 \mathrm{e}-8$ & 7.88750 & 0.253085 & 1.12234 & 5.64354 & 1.01837 & 0 & 0 & 0.2100 \\
\hline 9 & $1.59713 \mathrm{e}-7$ & 5.97718 & 0.379652 & 0.825954 & 0.857850 & 0 & 0 & 6.11295 & 0.2035 \\
\hline 10 & 1.28249 & 0.788528 & 1.19686 & 0.595343 & 0.772734 & 0.0263791 & 0.913011 & 1.77834 & 0.3538 \\
\hline 11 & $1.79254 \mathrm{e}-13$ & 11.76514 & 0.564683 & 0.905328 & 0.375291 & 0 & 0 & 2.47176 & 0.1360 \\
\hline 12 & \begin{tabular}{|l|}
0.0264789 \\
\end{tabular} & 1.10650 & 0.119784 & 0 & 0.497133 & 0 & 0 & 1.05946 & 0.2198 \\
\hline 13 & $1.31795 \mathrm{e}-8$ & 11.27609 & 2.044278 & 0.735657 & 0.594690 & 0 & 0 & 0.978506 & 0.0465 \\
\hline
\end{tabular}

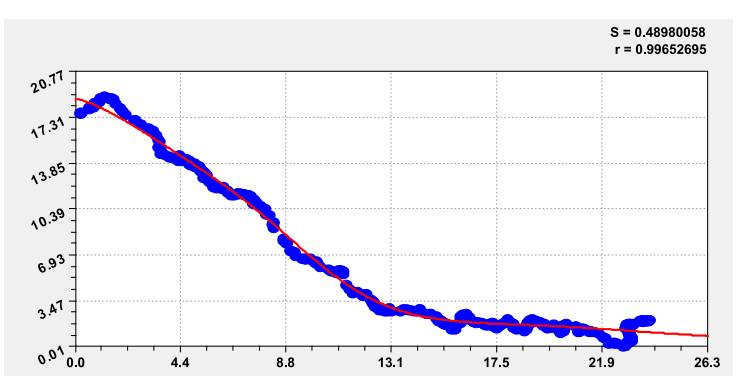

Двухчленный тренд

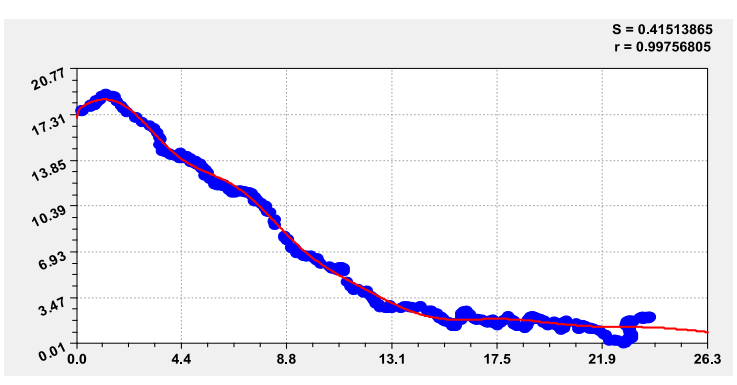

Двухчленный тренд и колебание

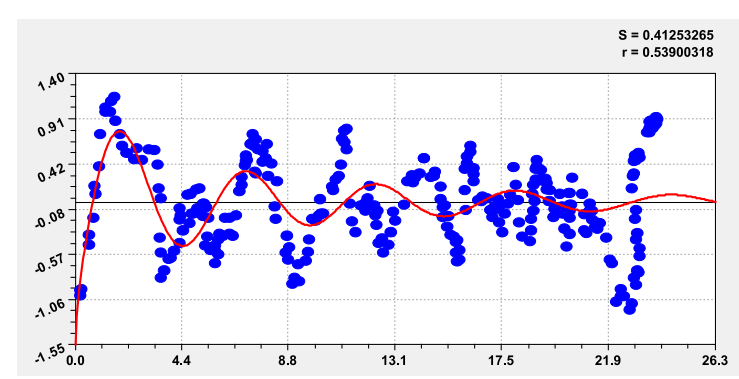

Колебание

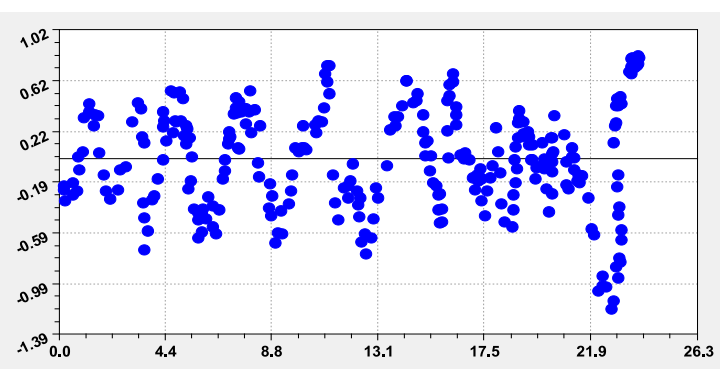

Остатки после трехчленной модели

Рис. 1. Графики влияния широть Север - Юг от истока до устья на долготу от левой точки русла реки Ировка

Идентификацией модели (1) было получено 18 вейвлетов долготы (табл. 3, рис. 2-4).

Первые три вейвлета дали коэффициента корреляции 0.9991. Первый член есть модифицированный закон Мандельброта [5], и он показывает экспоненциальный разворот русла на запад. Второй член по биотехническому закону [5] дает возбуждение рельефа на запад. Три колебания указывают на позитивное изменение ландшафта при- мерно до середины малой реки. А шестой член характерен для поведения устья малой реки Ировка.

Еще шесть членов показывают локальное изменение рельефа русла реки. Таким образом, географическая долгота вдоль русла малой реки изменяется, как и широта, волнообразно по асимметричным вейвлетам. На это влияет колеблющийся в плане стрежень русла малой реки Ировка между характерными точками малой реки. 
Таблица 3

Параметры (1) распределений по рангам долготы реки Ировка

\begin{tabular}{|c|c|c|c|c|c|c|c|c|c|}
\hline \multirow[t]{3}{*}{$\begin{array}{c}\text { Номер } \\
i\end{array}$} & \multicolumn{8}{|c|}{ Вейвлет $y_{i}=a_{1 i} x^{a_{2 i}} \exp \left(-a_{3 i} x^{a_{4 i}}\right) \cos \left(\pi x /\left(a_{5 i}+a_{6 i} x^{a_{7 i}}\right)-a_{8 i}\right)$} & \multirow{3}{*}{$\begin{array}{c}\text { Коэф. } \\
\text { корр. } \\
r\end{array}$} \\
\hline & \multicolumn{4}{|c|}{ Амплитуда (половина) колебания } & \multicolumn{3}{|c|}{ Полупериод колебания } & СдВиг & \\
\hline & $a_{1 i}$ & $a_{2 i}$ & $a_{3 i}$ & $a_{4 i}$ & $a_{5 i}$ & $a_{6 i}$ & $a_{7 i}$ & $a_{8 i}$ & \\
\hline 1 & 18.74005 & 0 & 0.0018915 & 1.31141 & 0 & 0 & 0 & 0 & 0.9991 \\
\hline 2 & $-3.54095 \mathrm{e}-14$ & 94.91149 & 150.10476 & 0.21629 & 0 & 0 & 0 & 0 & \\
\hline 3 & $1.44702 \mathrm{e} 6$ & 6.58267 & 20.25179 & 0.17525 & 3.00095 & .03474 & 040801 & 14536 & \\
\hline 4 & $7.26081 \mathrm{e}-7$ & 3.57383 & 0.00167623 & 1.62071 & 18.84378 & $8.39282 \mathrm{e}-5$ & 1.89187 & 1.5461 & 0.7776 \\
\hline 5 & 0.136962 & 0.711550 & 0.282787 & 0.558071 & 10.13446 & 0.0879591 & 0.572429 & 3.0931 & 0.5012 \\
\hline 6 & $e-7$ & 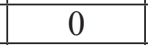 & $\overline{0411}$ & 1.02605 & 04.72321 & 0.00560603 & 1.04115 & 0.592858 & 0.7010 \\
\hline 7 & 0.00286152 & 1.02550 & $4.52966 \mathrm{e}-4$ & 1.68641 & 6.85528 & 0.00267353 & 0.82799 & 1.68637 & 0.5040 \\
\hline 8 & 1.02 & 15.84112 & 0.06 & 1.16178 & 1.94778 & 0 & 0 & 1.60019 & 0.2269 \\
\hline 9 & 2.475 & 80.52037 & 0.68 & 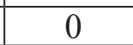 & 4.99899 & $2465 e-4$ & 841448 & 1.59807 & 0.4686 \\
\hline 10 & 0.0369682 & \begin{tabular}{|l|}
0.184497 \\
\end{tabular} & $2.28544 \mathrm{e}-4$ & 1.52781 & 23.39567 & $3.84322 \mathrm{e}-4$ & 1.86656 & 1.77078 & 0.5315 \\
\hline 11 & & 1.55214 & 0.025 & & \begin{tabular}{|l|l|}
8.72253 \\
\end{tabular} & 8.9322 & 1.33455 & 1.58790 & 0.4655 \\
\hline 12 & -5 & 2.05055 & 0.20 & 0.61 & 1.55638 & 0.0014 & 1.55734 & 3.09580 & 0.3152 \\
\hline 13 & $3.96597 \mathrm{e}-16$ & 7.66169 & 0.0185167 & 1.17166 & 7.65696 & 0.00346936 & 1.21366 & 13.37270 & 0.1871 \\
\hline 14 & & & & & 4.34034 & $332126 e-4$ & 1.13981 & 2.07944 & 0.2893 \\
\hline 15 & & 1.88212 & 959 & \begin{tabular}{|l|l|}
0.994927 \\
\end{tabular} & 1.38098 & $2.34393 \mathrm{e}-4$ & 1.04177 & 0.067522 & 0.1450 \\
\hline 16 & 0.00716105 & \begin{tabular}{|l|}
0.249398 \\
\end{tabular} & $5.24321 \mathrm{e}-4$ & 1.52331 & 5.46810 & 0.966844 & 0 & 0 & 0.1184 \\
\hline 17 & $4.58922 \mathrm{e}-63$ & 37.51718 & 0.11 & 1.21796 & 1.67233 & 0 & 0 & 2.19317 & 0.3101 \\
\hline 18 & $6.0678 \mathrm{e}-34$ & 19.30668 & 0.118273 & 1.05796 & \begin{tabular}{|l|}
4.17660 \\
\end{tabular} & \begin{tabular}{|l|l|}
0.00108311 \\
\end{tabular} & 1.22631 & 7.95796 & 0.3836 \\
\hline
\end{tabular}
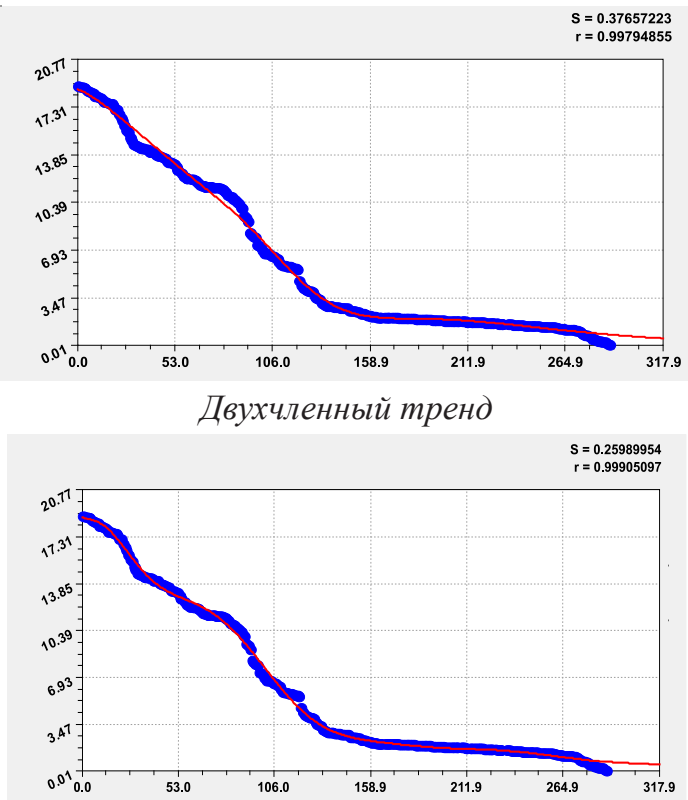

Двухчленный тренд и колебание

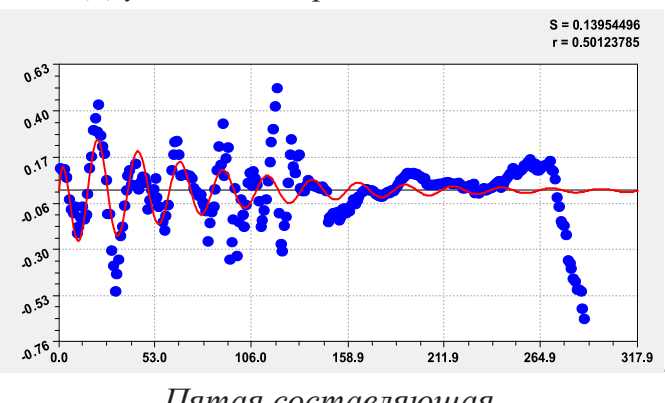

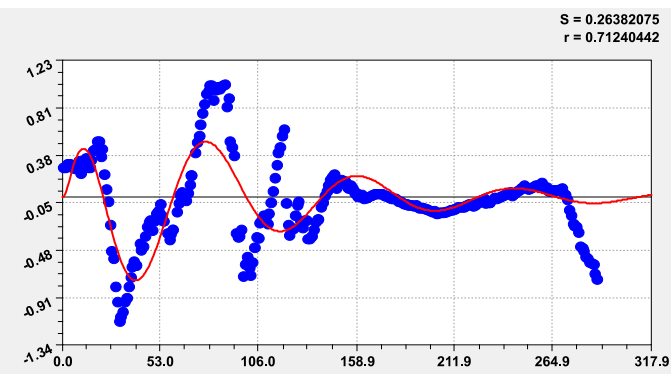

Первое колебание

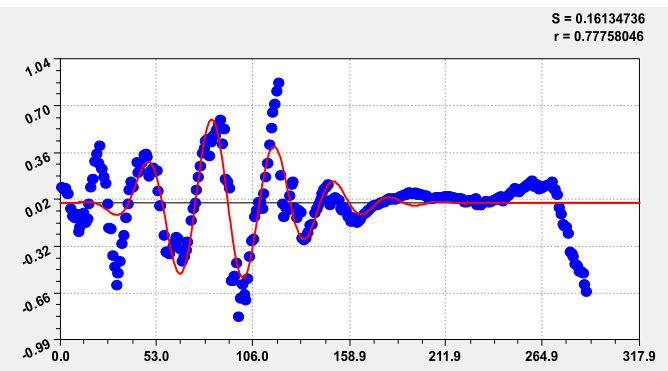

Четвертая составляющая

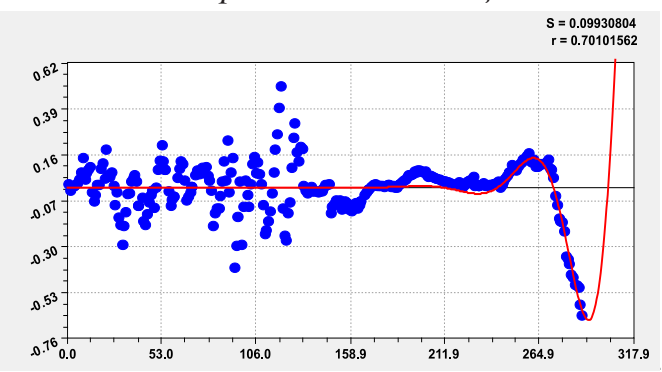

Шестая составляюшая

Рис. 2. Графики моделей распределения по рангам долготы реки Ировка 

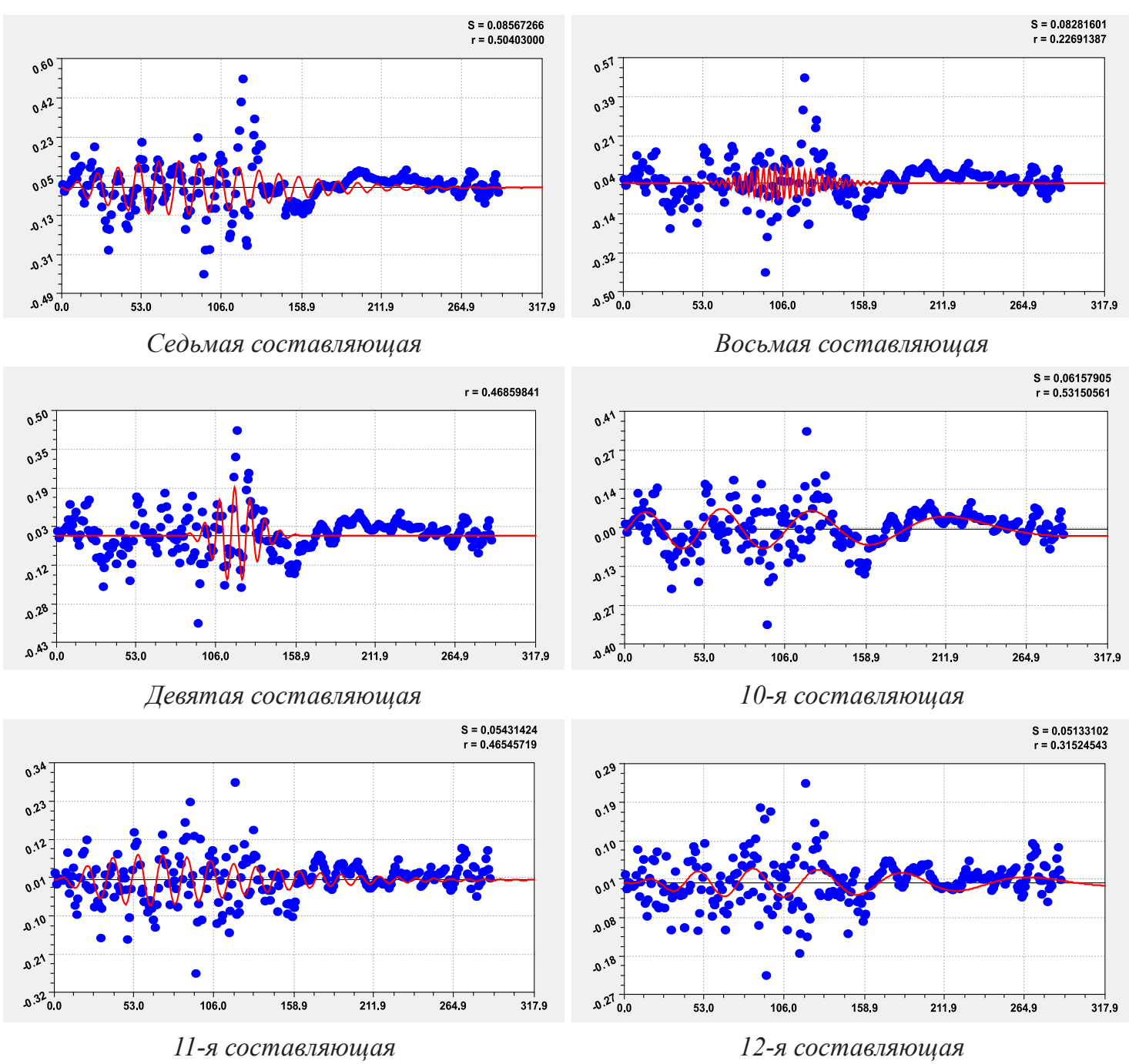

Рис. 3. Графики дополнительных моделей распределения долготы реки Ировка по рангам

На рис. 4 появляются вдоль реки несколько коротких вейвлетов.

После 18-го члена общей модели (1) процесс квантования вейвлетов сильно затрудняется. Это означает, что погрешность измерений дальше не позволяет получать высокую квантовую определенность. Для продолжения квантования всей речной системы нужно только уменьшить погрешность измерений. Рельеф получает квантовую запутанность только при значительном антропогенном влиянии. По-видимому, это замечательное математическое свойство стабильности динамики рельефа заключается в его естественной эволюции за очень продолжительное время (без влияния человека столетиями).

Остатки от 18-го колебания после рангового распределения долготы дали высокую погрешность моделирования $33.36 \%$ ближе к устью на точке с рангом 250 (табл. 1).
В этом месте происходит поворот малой реки Ировка на восток. По местной широте намного большая погрешность была получена в истоке Ировки (бесконечность), поэтому верховье реки летом пересыхает именно из-за изменений высоты в зависимости от широты и долготы.

По интервалам 0,1 погрешность распределилась так (по модулю): при нуле 3 шт. (1.03\%); 0-0,1\% всего 37 шт. (12,76\%); $0,1-0,2 \% \quad 29$ шт. $(10,00 \%) ; \quad 0,2-0,3 \%$ 33 шт. (11,38\%); 0,3-0,4\% 24 шт. (8.28\%); 0,4-0,5\% 22 шт. $(7,59 \%)$ и т.д. По крупным интервалам: 0-1,0\% всего 203 шт. (70\%); от 0 до $5 \%$ распределяются 277 характерных точек русла, что равна 95,52\% от 290 характерных точек. Норму погрешности измерений и моделирования примем $5 \%$, тогда оставшиеся $290-277=13$ точек русла требуют отдельного рассмотрения. 

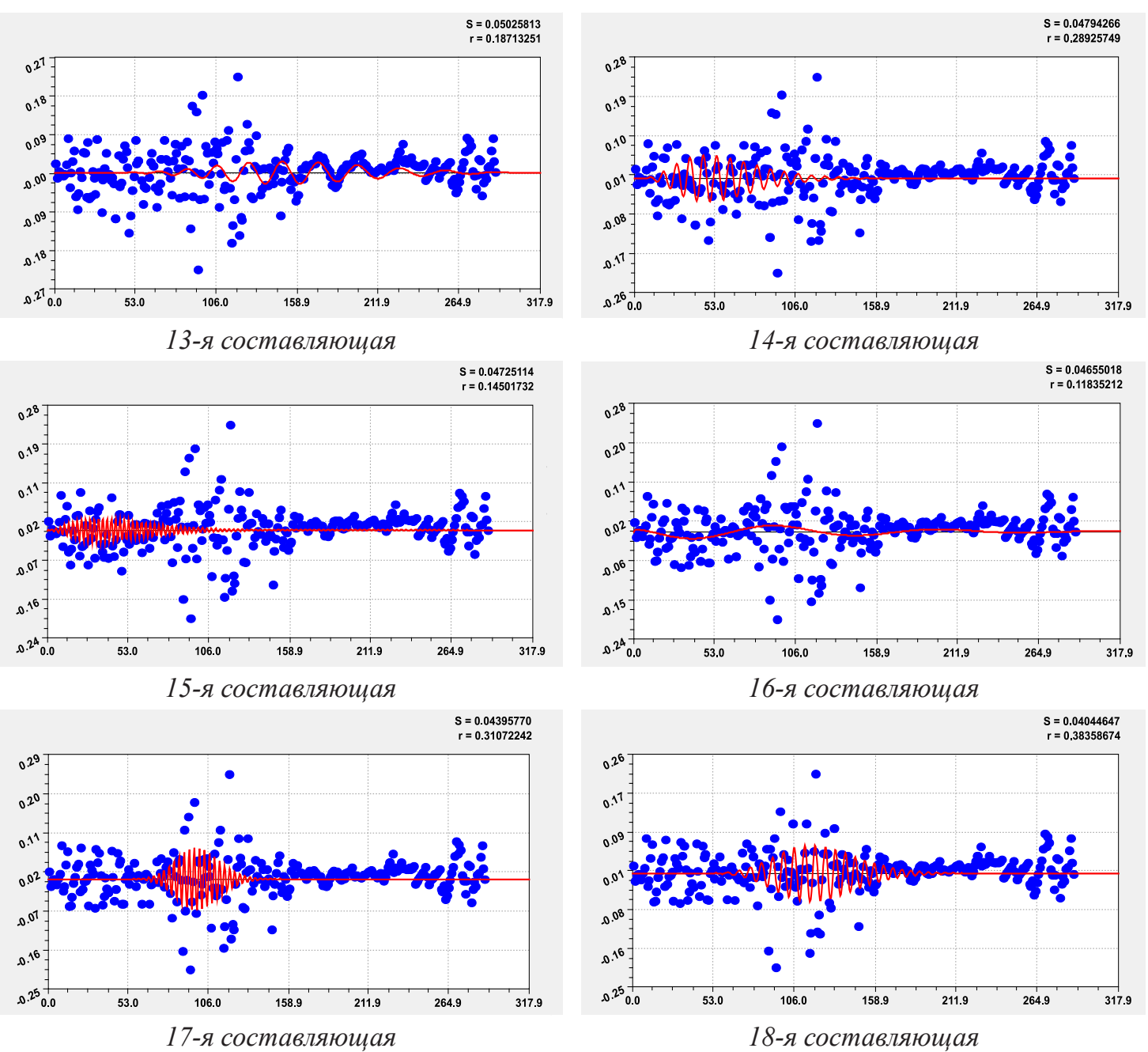

Рис. 4. Графики последних моделей распределения долготы реки Ировка по рангам

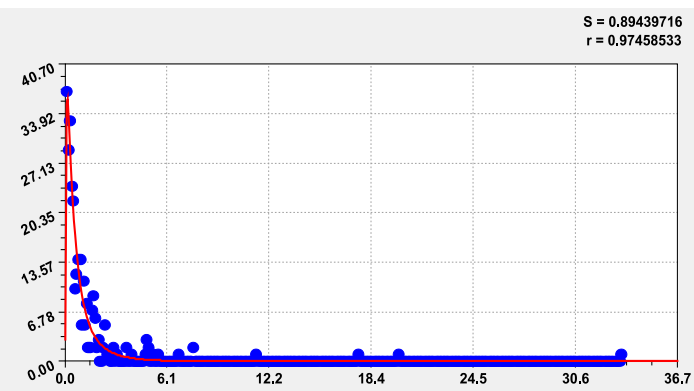

Двухчленный тренд

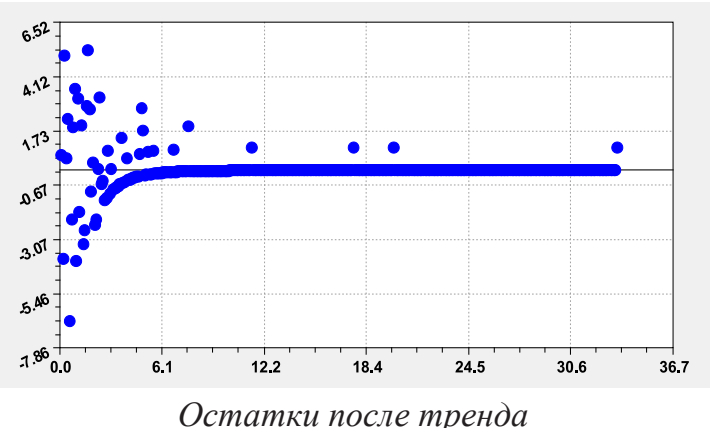

Остатки после тренда

Рис. 5. Графики распределения погрешности моделирования долготы реки Ировка

На рис. 5 приведен график распределения погрешности модели по формуле

$$
\begin{gathered}
n=3.02237 \exp \left(-0.60656 \Delta_{0.1}\right)+ \\
+943.91958 \Delta_{0.1}^{0.76971} \exp \left(-4.85636 \Delta_{0.1}{ }^{0.49132}\right) .
\end{gathered}
$$

По остаткам от тренда (2) наибольший разброс наблюдается при погрешности мо- делирования до $5 \%$. По второй части этой формулы происходит резкий всплеск до $3 \%$.

\section{Заключение}

Моделирование распределений приведенной к данной местности малой реки долготы позволило получить 18 составляющих 
общей формулы вейвлета с распределением погрешности моделирования в основном до $5 \%$. При этом особое значение приобретает волновое изменение местной широты от Севера на Юг (было получено 25 членов) и местной долготы (19 членов рангового распределения) на примере от истока до устья малой реки Ировка). В итоге географические координаты малой реки в плане позволяют математически описать рельеф русла.

Первые три члена модели (1) для долготы дали адекватность по коэффициенту корреляции 0.9991. Уровень адекватности более 0.99 , и он относится к сильнейшей факторной связи. Остальные 15 членов (1) увеличивают адекватность до 1. Долгота малой реки Ировка по космоснимкам позволяет получить высокую определенность процесса квантования вейвлетов.

По крупным интервалам погрешность моделирования после 18-го члена распределяется так: 0-1,0\% всего 203 шт. (70\%); от 0 до $5 \%$ распределяются 277 характерных точек русла, что равно 95,52\% от 290 характерных точек. Норму погрешности измерений и моделирования примем $5 \%$, тогда оставшиеся $290-277=13$ характерных точек русла малой реки Ировка требуют отдельного рассмотрения.

\section{Список литературы / References}

1. Садков С.А. Исследование морфологической структуры равнин с развитием просадочных процессов на осно- ве подходов математической морфологии ландшафтов // Геоморфология. 2019. № 1. С. 48-58. DOI: $10.31857 /$ S0435-42812019148-58.

Sadkov S.A. Morphological structure of plains subject to ground Subsidence on the basis of mathematical morphology of landscapes approach // Geomorfologiya. 2019. № 1. P. 4858 (in Russian).

2. Харченко С.В. К вопросу о применении гармонического анализа при количественной характеристике рельефа // Геоморфология. 2017. № 2. С. 14-24. DOI: 10.15356/0435-4281-2017-2-14-24.

Kharchenko S.V. Application of harmonic analysis for the quantitative description of earth surface topography // Geomorfologiya. 2017. № 2. P. 14-24 (in Russian).

3. Рулев А.С., Юферев В.Г. Математическое и геоморфологическое моделирование эрозионных ландшафтов // Геоморфология. 2016. № 3. C. 36-45. DOI: 10.15356/0435-4281-2016-3-36-45.

Rulev A.S., Yuferev V.G. Mathematical and geomorphological modeling of the erosion landscapes // Geomorfologiya. 2016. № 3. P. 36-45 (in Russian).

4. Карты высот, уклонов. [Электронный ресурс]. URL: http://votetovid.ru/\#56.201192,48.95536,17z,51v301 (дата обращения: 02.12.2019).

Maps of heights, slopes. [Electronic resource]. URL: http:// votetovid.ru/\#56.201192,48.95536,17z,51v301 (date of access: 02.12.2019) (in Russian).

5. Mazurkin P.M. Wavelet Analysis Statistical Data. Advances in Sciences and Humanities. 2015. Vol. 1. No. 2. P. 3044. DOI: 10.11648/j.ash.20150102.11.

6. Георгиева Я.О., Мазуркин П.М. Сравнение земельного покрова по берегам вдоль реки Ировка по спутниковым снимкам // Международный студенческий научный вестник. 2019. № 6. [Электронный ресурс]. URL: http://eduherald.ru/ ru/article/view?id=19812 (дата обращения: 27.12.2019).

Georgieva Ya.O., Mazurkin P.M. Comparison of land cover along the banks of the Irovka River by satellite imagery // Mezhdunarodnyy studencheskiy nauchnyy vestnik. 2019. № 6. [Electronic resource]. URL: http://eduherald.ru/ru/article/ view? $\mathrm{id}=19812$ (date of access: 27.12.2019) (in Russian). 